\title{
Observation of Electrohydrodynamic Flow through a Pore in Ion-Exchange Membrane
}

\author{
Ayako Yano, Kentaro Doi, and Satoyuki Kawano
}

\begin{abstract}
Liquid flows driven by electric force is known as electrohydrodynamics (EHD). EHD flows are expected to be applied to micropumps, microactuators, and mixing devices. However, it is known that conventional EHD flows require at least tens of volts of the applied voltage. In this study, a novel device is developed to generate an EHD flow under a constant current condition with a few voltages. An ion-exchange membrane that has a small pore is set in a reservoir to separate the flow path of anion and cation. The reservoir is filled with $\mathrm{NaOH}$ aqueous solution and a constant electric current is applied across the membrane. When the cross sectional area of the ion-exchange membrane is 200 times larger than that of the pore, EHD flows observed in the pore become faster than those in previous studies. The maximum value of the flow velocity reaches $3 \mathrm{~mm} / \mathrm{s}$ by applying a constant current of $0.8 \mathrm{~mA}$.
\end{abstract}

Index Terms-Electrohydrodynamics, ion exchange membrane, microfluidics, visualization.

\section{INTRODUCTION}

Recently nanofabrication techniques have made rapid progresses. In nano-scaled fluidic devices, ion transports strongly affect the liquid flows that often result in complicated flow patterns [1], [2]. Such peculiar flows seem to exceed our understanding by means of conventional ion transport theories [3]. For example, a liquid flow is induced due to high concentrations of electrolyte ions near a charged wall surface when an electric field is externally applied in the solutions as shown in Fig. 1(a). This kind of flow is known as electroosmotic flow (EOF) [2], [3], as schematically shown Fig. 1(b). EOFs are one of the hottest topics in micro/nanofluidics and further understanding is required. A charged wall surface in electrolyte solution attracts counter ions and it forms electric double layer (EDL). Particularly, when a distance between two channel wall surfaces becomes closer, EDLs overlap with each other as shown in Fig. 1(c). In such a narrow channel, electroneutrality is broken and dominant ion transports drive liquid flows. As mentioned above, electrically polarized conditions are inevitable to induce liquid flows by externally applied electric force.

In recent, novel technologies to manipulate electrophoretic behavior of single molecules [4]-[6], charged particles [7], and allergens [8] by applying EOFs have attracted much attention. Charged molecules, such as deoxyribonucleic acid (DNA) and ribonucleic acid (RNA) in electrolyte solutions are electrically driven by external electric fields. Controlling

Manuscript received September 18, 2014; revised December 9, 2014.

Ayako Yano, Kentaro Doi, and Satoyuki Kawano are with the Department of Mechanical Science and Bioengineering, Graduate School of Engineering Science, Osaka University, Toyonaka, Osaka 560-8531, Japan (e-mail: kawano@me.es.osaka-u.ac.jp). the transport velocity is crucial to clearly detect a single molecule in extremely narrow spaces, such as nanoslits and nanopores. To do so, a deep understanding of responses of electrolyte ions and liquid flow are inevitable [9].

Electrolyte ions in solutions cause to induce a liquid flow when externally electric force is applied and then, the ions drag surrounding fluids. This flow is called as electrohydrodynamic (EHD) flow [10]-[13]. The idea of EHD flows was based on a pioneering work by Melcher and Taylor [10]. Following them, Saville [11] and his coworkers [12], [13] developed the theoretical basis and optically observed the phenomenon. EHD flows are expected to be applied to micropumps and mixing techniques [14]. However, high voltages at least several tens of volts are usually required to make EHD flows. Bhaumik et al. [14] developed a low voltage driving micropump. But, its output flow velocity was lower than the conventional ones, and the flow rate was not enough. For example, they applied AC fields $\left(15-40 \mathrm{~V}_{\mathrm{pp}}\right.$; 5-500 Hz) across the two parallel electrodes. By applying 15 $\mathrm{V}_{\mathrm{pp}}$, the velocity of the generated EHD flow resulted in 107 $\mu \mathrm{m} / \mathrm{s}$, in which the distance between the electrodes was set to $600 \mu \mathrm{m}$.

In this study, we tackle to develop a novel device to investigate EHD flows, which works with moderate currents and voltages. As schematically shown in Fig. 1(d), an EHD flow passing through a small pore placed in an ion-exchange membrane is optically and electrically measured. Applying an electric current along the channel axis, both the anion and cation are separately attracted to the counter electrodes. In a case using an anion-exchange membrane, anions can pass through all cross-sectional areas including a pore region, but cations are limited to pass through a pore in the membrane. In the experimental system, due to a large difference between a cross-sectional area of anion-exchange membrane and its pore, highly concentrated cations in the pore drive a strong EHD flow. As a result, our device can make a significantly fast EHD flow compared to previous results [14]. Further progresses based on the present results are expected to be developed not only for translocation velocity control of single molecules but also for arbitrary molecular pattern fabrications [15].

\section{EXPERIMENTAL METHODOLOGY}

\section{A. Design of Experimental Device}

Fig. 1(e) shows a schematic illustration of the present experimental device. An ion-exchange membrane with a pore and a pair of helical shaped Au electrodes is immersed in a reservoir made of polydimetylsiloxane (PDMS). An anoin-exchange membrane (Neosepta ${ }^{\circledR}$ A-0325, ASTOM 
Co., Ltd., Tokyo, Japan) was employed in this experiment. The experimental device was designed to set the cross-sectional area of ion-exchange membranes 200 times larger than that of the pore in the membrane, where the ion exchange membranes had a diameter of $18.0 \mathrm{~mm}$. A $1 \mathrm{~mm} \times 1$ $\mathrm{mm}$ rectangular cross-section pore was $3 \mathrm{~mm}$ in length. This channel was penetrated in the ion-exchange membrane. The system consisted of two different-sized cylindrical chambers: 14.4 and $18.4 \mathrm{~mm}$ in diameter. A glass cylindrical chamber (18.0 $\mathrm{mm}$ in diameter and $10 \mathrm{~mm}$ in length) and a spacer (18.0 $\mathrm{mm}$ in diameter and $5 \mathrm{~mm}$ in length) were placed in both sides of the reservoir to fix an ion-exchange membrane. A $0.83 \mu \mathrm{m}$ diameter polystyrene particle (Estapor ${ }^{\circledR}$, MERCK KGaA Co., Ltd., Dermstadt, Germany) was employed as a tracer particle. A 10 volume percent solution of the polystyrene particle was diluted in pure water to 0.1 volume percent, and $200 \mu \mathrm{L}$ of it was added in $3.6 \mathrm{~mL}$ of a $0.1 \mathrm{M} \mathrm{NaOH}$ solution for visualization of EHD flows.

\section{B. Experimental Method}

Applying a constant current or a constant voltage, electric signals and flow velocities were measured. Behavior of tracer particle in the pore was observed by using a microscope with a high speed camera (VW-9000 Keyence). A constant current of $0.8 \mathrm{~mA}$ or a constant voltage of $2.2 \mathrm{~V}$ was applied in the solution on the Au electrodes by a potentiostat (VersaSTAT4 AMTEK). Trajectories of the particles were traced at a frame rate of $125 \mathrm{fps}$ and the flow velocity was analyzed. The time response of electric signals was simultaneously measured with a sampling rate of $200 \mathrm{~Hz}$. After the reservoir was filled with the solution, at $t=5,30,55$, and $80 \mathrm{~min}$, electric force was applied to observe EHD flows. Before the measurements, the electrodes were shorted and the solution was sufficiently stirred to maintain equilibrium.

\section{RESULTS AND DISCUSSION}

\section{A. Confirmation of the Experimental Condition}

Polystyrene particles are usually known to be negatively charged in aqueous solutions and we need to clarify the degree of electric force on polystyrene particles. A 0.1 volume percent solution of the tracer particle was injected near the pore. After the solution was equilibrated, the inside of the pore was observed from the upper side, applying electric potentials up to $5 \mathrm{~V}$ for $60 \mathrm{~s}$. As a result, any effective acceleration of the particles by the electric fields could not be found, even though the Brownian motion was clearly observed. Thus, it was concluded that electrophoretic force on the tracer particle was negligibly weak to the applied voltage less than $5 \mathrm{~V}$ in this system.

\section{B. Anion-Exchange Membrane}

Fig. 2 shows some typical results of the time transition of voltage difference between the electrodes when the constant current of $0.8 \mathrm{~mA}$ was applied from $t=5$ to $35 \mathrm{~s}$. The velocity response of tracer particles was observed in the pore in the anion-exchange membrane. Here, the positive direction of the velocity means a direction along which cations are driven by electrophoresis. As shown in Fig. 2(a), the flow velocity immediately rose up to $1000 \mu \mathrm{m} / \mathrm{s}$ when the electric current was applied at $t=5 \mathrm{~s}$ and then, asymptotically approached zero. In Fig. 2(b), two peaks were observed in the velocity response and there seemed to include different time scales. The velocity reached $1800 \mu \mathrm{m} / \mathrm{s}$ at $t=6 \mathrm{~s}$. After that, in the decay process, the second spike was observed at $t=18 \mathrm{~s}$. The time constant of the first peak was longer than that of the second one. Fig. 2(c) shows three peaks over $1000 \mu \mathrm{m} / \mathrm{s}$. The values were 1000,1400 , and $3000 \mu \mathrm{m} / \mathrm{s}$ at $7.5,8.5$, and $15.5 \mathrm{~s}$, respectively. The velocity of $3000 \mu \mathrm{m} / \mathrm{s}$ was the maximum in this experiment. For the reference, Fig. 2(d) presents a result in which no effective response was observed, even though the electric current was applied. Weak peaks near $400 \mu \mathrm{m} / \mathrm{s}$ were often observed at $t=24$ and $27 \mathrm{~s}$.
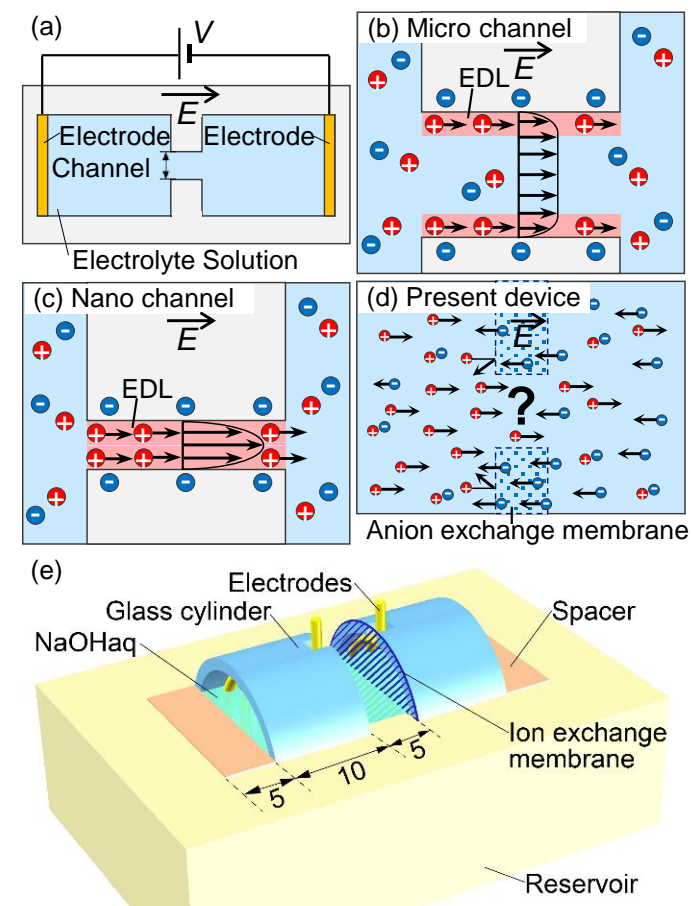

Unit : $\mathrm{mm}$

Fig. 1. Schematic illustrations: (a) whole structure of the experimental system, (b) electroosmotic flow (EOF) in microchannel, (c) EOF in nanochannel, (d) EHD flow in the present system, and (e) design of the present experimental device.

Most of the results indicate that strong liquid flows are induced when an electric current is applied. Following that, the transient flow velocity is reduced to zero. The reason is that the electric current controls the surface charge of the electrodes; in the same time, almost uniform electric fields expand in the liquid; successively, electrolyte ions respond to the field. Then, surfaces of the electrodes are screened by ions that quickly move very near the electrodes. As shown in Fig. 2, the voltage response is transient and asymptotically approaches constant values near 2.0-2.5 V. In this period, the behavior of electrolyte ions is drastically modulated to form EDLs and to maintain the constant current condition. That is, ion transports are also excited everywhere in the solution, not only near the electrode surfaces. Gradually forming EDLs, the solution achieves a steady condition. In this process, the current path of cations is limited and highly concentrates in 

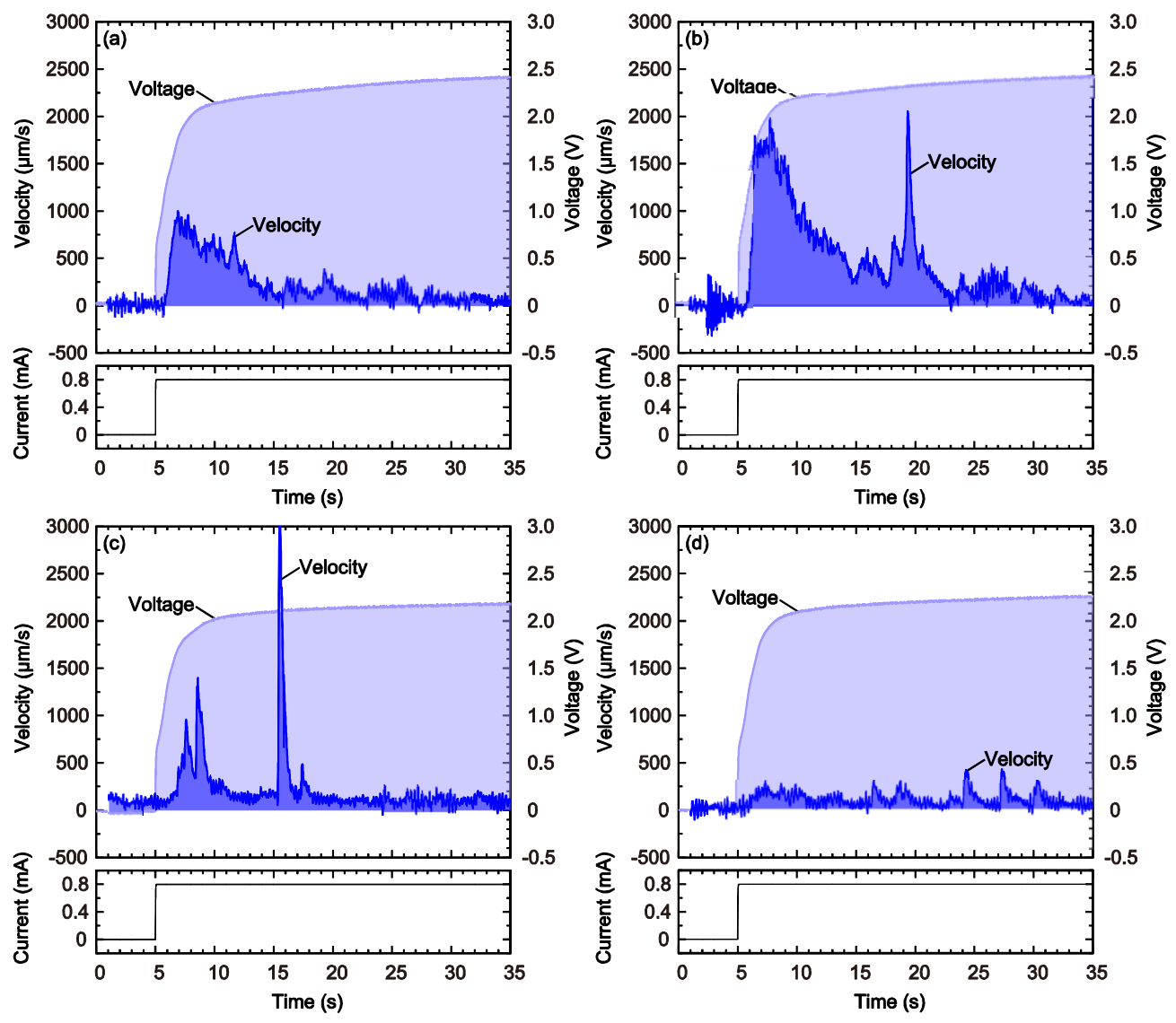

Fig. 2. Voltage and velocity responses observed in the pore placed in an anion-exchange membrane, where an electric current is applied at $t=5 \mathrm{~s}$. According to transient voltage responses, the velocity presents a variety of responses: (a) a single peak and slow decay to zero, (b) two peaks with different time constants, and (c) two peaks of spike like responses, referring to the case of (d) no prominent responses in the velocity.

the pore. This is a reason why strong liquid flows are frequently induced along the cation flow direction during the transient response of the voltage difference. In the present system, the constant electric current is maintained by electrolysis of water in the alkaline solution. Then, $\mathrm{Na}^{+}$ions, which are finite in the solution, may not be an effective electric carrier to the constant current. Therefore, the velocity response due to the $\mathrm{Na}^{+}$transport results in transient. This is the essence of the present EHD flow. In this study, we succeeded to experimentally observe the EHD flow passing through a millimeter-sized pore placed in an anion-exchange membrane. Although nanometer-sized channels are usually required to maintain electrically polarized conditions in liquid phase as shown by the mechanism of EOF (Fig. 1(a), (b)), our device supposes another method to break the electroneutrality in millimeter-sized spaces.

\section{EHD Generation by Applying Constant Voltages}

It was found that EHD flows could be generated by applying a constant electric current and that the direction of observed liquid flows corresponding to that of cation transport. Additionally, we conducted another experiment to clarify that EHD flows could also be induced by applying constant voltages. In Fig. 2, at the steady states, the voltage response seemed to be settled at 2.0-2.5 V. Referring to this result, a constant voltage difference of $2.2 \mathrm{~V}$ was applied on the electrodes. Fig. 3 shows the response of flow velocity and electric current. The results show a similar velocity response with Fig. 2. At $t=5 \mathrm{~s}$, the electric potential was externally applied and then, a spike signal of electric current could be observed. Successively, a liquid flow immediately increased and decayed to zero. In the velocity response, many spikes seemed to be included. The mechanism of the EHD flow can be explained in the same manner as the constant current response. To maintain a constant voltage difference between the electrodes, the electric current is controlled against the ionic current in the solution. Thus, the electric current reflects the behavior of ions. According to the ionic response, liquid flow was also induced due to highly concentrated $\mathrm{Na}^{+}$ions near the pore. Consequently, it was found that both constant current and constant voltage measurements were effective to induce the EHD flows. This result is explained by a theoretical model of liquid flow affected by externally applied electric stresses, which will be reported elsewhere.

\section{Effects of Membrane Treatments on Electric Current}

To investigate the effects of membrane treatment procedures on the electric current response, we compared three treatment processes. Fig. 4 shows current response of each membrane with an applied voltage of $2.2 \mathrm{~V}$. In each condition, the electric current response was measured five times for each two membrane and the average values were plotted. The first method is ultrasonic cleaning in pure water. An anion-exchange membrane was immersed in pure water in an ultrasonic cleaner for 10 min twice. In the second method, the membrane was put in $\mathrm{NaOH}$ solution for $2 \mathrm{~h}$ after the ultrasonic cleaning. In the third method, after the ultrasonic cleaning, the membrane was set in the reservoir and the electric current of $0.8 \mathrm{~mA}$ and $-0.8 \mathrm{~mA}$ were applied to the membrane for $1 \mathrm{~h}$ each direction. 


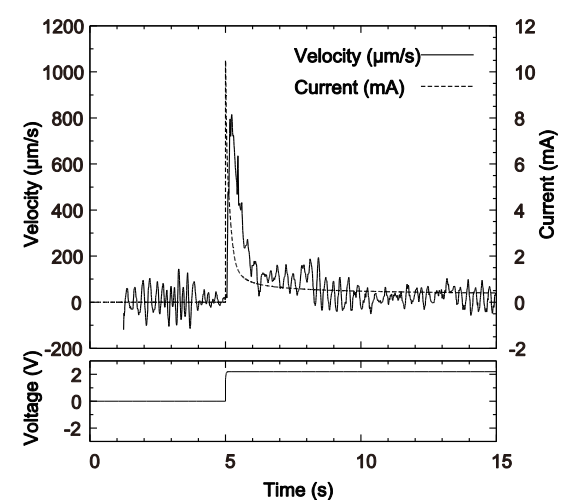

Fig. 3. Velocity and electric current responses, applying a constant voltage of $2.2 \mathrm{~V}$ at $t=5 \mathrm{~s}$. A single sharp peak was found immediately when the voltage was applied.

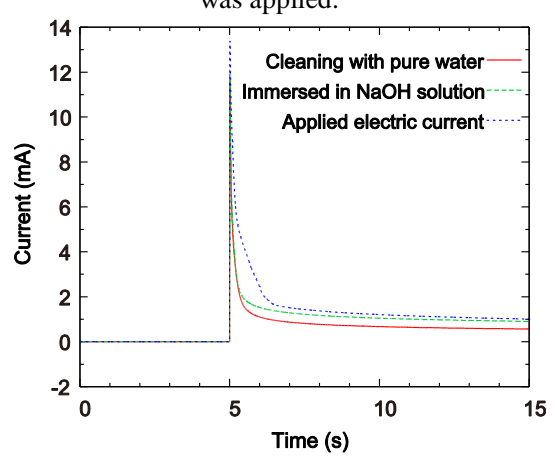

Fig. 4. Electric current response with respect to different conditions of the membrane.

In Fig. 4, peaks, relaxation times, and constant values at the steady current state are compared between the treated membranes mentioned above. The relaxation time $\tau$ of electrophoretic mobility $\mu$ can be written as $\mu=z e \tau / m$, where $z$ is the valence number, $e$ is the elementary charge, $m$ is the mass. A relationship between the conductance $\sigma$ and $\mu$ is represented by $\sigma=\rho \mu$, where $\rho$ is a charge density. That is, $\sigma$ is proportional to $\tau$. The longer $\tau$ results in the better $\sigma$. The anion-exchange membrane was usually kept in $3 \% \mathrm{NaCl}$ aqueous solution. When the membrane was cleaned in pure water, the conductance tended to decrease because additional ions were removed. Before applying external fields, the long immersion time of the membrane in $\mathrm{NaOH}$ solution also improved the $\sigma$, because the gradation of electrolytes was equilibrated. As a result, the $\sigma$ of membranes was improved. Under the applied current condition, ionic current paths actually penetrated the membrane and the $\sigma$ achieved more preferable conditions. In this case, the $\tau$ became the largest among the treatments. This result also indicates that the electric current responses actually reflect the ionic current responses in the solution. This means that the non-equilibrium response of ions in the membrane is actually recognized, even if the membrane is positioned apart from the electrodes. The time transitions of flow velocities, as shown in Fig. 2 and Fig. 3 , are optically and electrically confirmed to be caused by the ionic motions.

\section{CONCLUSION}

In this study, a novel device was developed to observe an EHD flow. Placing a 1-mm scale pore in an anion-exchange membrane, an electrically polarized condition could be generated under a constant current condition. Strong EHD flows were generated in the pore and the maximum flow velocity achieved $3 \mathrm{~mm} / \mathrm{s}$. It was confirmed that the EHD flow actually induced along the cation flow direction in a $0.1 \mathrm{M}$ $\mathrm{NaOH}$ solution. That is, we succeeded to locally visualize the break of electroneutrality in the solution. In the flow velocity responses, the voltage difference asymptotically approached a steady value near 2.0-2.5 V. Furthermore, similar EHD flows could be observed by applying a constant voltage of $2.2 \mathrm{~V}$.

In the near future, theoretical results will be reported elsewhere to deeply understand the EHD flow in which a liquid flow is induced by ionic responses according to electric field formations [9].

\section{REFERENCES}

[1] W. Guan, R. Fan, and M. A. Reed, "Field-effect reconfigurable nanofluidic ionic diodes," Nat. Commun., vol. 2, no. 506, pp. 1-8, 2011.

[2] H. Daiguji, P. Yang, and A. Majumdar, "Ion transport in nanofluidic channels," Nano Lett., vol. 4, pp. 137-142, 2004.

[3] R. B. Schoch, J. Han, and P. Renaud, "Transport phenomena in nanofluidics," Review of Modern Physics, vol. 80, pp. 839-883, July-September 2008.

[4] S. Uehara, H. Shintaku, and S. Kawano, "Electrokinetic flow dynamics of weakly aggregated $\lambda$ DNA confined in nanochannels," Trans. ASME., J. Fluids Eng., vol. 133, p. 12603, 2011.

[5] T. Yasui et al., "DNA manipulation and separation in sublithographic-scale nanowire array," ACS Nano, vol. 7, pp. 3029-3035, 2013.

[6] W. Qian et al., "Theoretical Study of the Transpore velocity control of single-stranded DNA," Int. J. Mol. Sci., vol. 15, pp. 13817-13832, 2014.

[7] N. Yukimoto et al., "Tracking single-particle dynamics via combined optical and electrical sensing," Sci. Rep., vol. 3, no. 1855, pp. 1-7, 2013.

[8] C. Kawaguchi et al., "Electrical Detection of Single Pollen Allergen Particles Using Electrode-Embedded Microchannels," J. Phys. Cond. Matter, vol. 24, p. 164202, 2012.

[9] K. Doi et al., "Nonequilibrium ionic response of biased mechanically controllable break junction (MCBJ) electrodes," J. Phys. Chem. C, vol. 118, pp. 3758-3765, 2014

[10] J. R. Melcher and G. I. Taylor, "Electrohydrodynamics: A review of the role of interfacial shear stresses," Annu. Rev. Fluid. Mech., vol. 1, pp. 111-146, 1969.

[11] D. A. Saville, "Electrohydrodynamics: The Taylor-Melcher leaky dielectric model," Annu. Rev. Fluid. Mech., vol. 29, pp. 27-64, 1997.

[12] W. D. Ristenpart, I. A. Aksay, and D. A. Saville, "Assembly of colloidal aggregates by electrohydrodynamic flow: Kinetic experiments and scaling analysis," Phys. Rev. E, vol. 69, p. 021405, 2004.

[13] W. D. Ristenpart, I. A. Aksay, and D. A. Saville, "Electrohydrodynamic flow around a colloidal particle near an electrode with an oscillating potential," J. Fluid. Mech., vol. 575, pp. 83-109, 2007.

[14] S. K. Bhaumik, R. Roy, S. Chakraborty, and S. DasGupta, "Low-voltage electrohydrodynamic micropumping of emulsions," Sens. Aactuat. B: Chemical, vol. 193, pp. 288-293, 2014.

[15] K. Doi, H. Takeuchi, R. Nii, S. Akamatsu, T. Kakizaki, and S. Kawano, "Self-assembly of $50 \mathrm{bp}$ poly(dA) p poly(dT) DNA on highly oriented pyrolytic graphite via atomic force microscopy observation and molecular dynamics simulation," J. Chem. Phys., vol. 139, p. 085102, 2013.

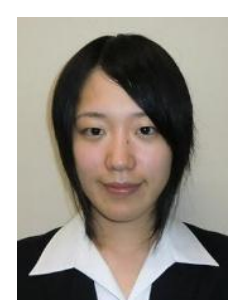

Ayako Yano is presently studying for master degree in Graduate School of Engeneering Science, Osaka University. She is supervised by Prof. Satoyuki Kawano. She received her bachelor of engineering from National Institution for Academic Degrees and University Evaluation in 2013. Her research topic is developing a novel device to generate an electrichydrodynamic flow under a constant voltage condition. 